\title{
A SHARP INTEGRAL HARDY TYPE INEQUALITY AND APPLICATIONS TO MUCKENHOUPT WEIGHTS ON R
}

\author{
Eleftherios N. Nikolidakis \\ National and Kapodistrian University of Athens, Department of Mathematics \\ Panepistimioupolis, GR 157 84, Athens, Greece; lefteris@math.uoc.gr
}

\begin{abstract}
We prove a generalization of a Hardy type inequality for negative exponents valid for non-negative functions defined on $[0,1)$. As an application we find the exact best possible range of $p$ such that $1<p \leq q$ such that any non-decreasing $\phi$ which satisfies the Muckenhoupt $A_{q}$ condition with constant $c$ upon all open subintervals of $[0,1)$ should additionally satisfy the $A_{p}$ condition for another possibly real constant $c^{\prime}$. The result have been treated in [9] based on [1], but we give in this paper an alternative proof which relies on the above mentioned inequality.
\end{abstract}

\section{Introduction}

During his efforts to simplify the proof of Hilbert's double series theorem, Hardy [5] first proved in 1920 the most famous inequality which is known in the literature as Hardy's inequality (see also [8], Theorem 3.5). This is stated as

Theorem A. If $p>1, a_{n}>0$, and $A_{n}=a_{1}+a_{2}+\cdots+a_{n}, n \in \mathbf{N}$, then

$$
\sum_{n=1}^{\infty}\left(\frac{A_{n}}{n}\right)^{p}<\left(\frac{p}{p-1}\right)^{p} \sum_{n=1}^{\infty} a_{n}^{p} .
$$

Moreover, inequality (1.1) is best possible, that is, the constant on the right side cannot be decreased.

In 1926, Copson generalized in [2] Theorem A by replacing the arithmetic mean of a sequence by a weighted arithmetic mean. More precisely, he proved the following

Theorem B. Let $p>1, a_{n}, \lambda_{n}>0$, for $n=1,2, \ldots$ Further, suppose that $\Lambda_{n}=\sum_{i=1}^{n} \lambda_{i}$ and $A_{n}=\sum_{i=1}^{n} \lambda_{i} a_{i}$. Then

$$
\sum_{n=1}^{\infty} \lambda_{n}\left(\frac{A_{n}}{A_{n}}\right)^{p} \leq\left(\frac{p}{p-1}\right)^{p} \sum_{n=1}^{\infty} \lambda_{n} a_{n}^{p}
$$

where the constant involved in (1.2) is best possible.

In [2], Copson proves also a second weighted inequality which as Hardy noted in [6] can be derived from Theorem B. From then and until now several generalizations have been given of the above two inequalities. The first one is given by Hardy and Littlewood who generalized in a specific direction Theorem 1.2 (see [7]). This was generalized further by Leindler in [12], and by Nemeth in [15]. Also in [14] one can see further generalizations of Hardy's and Copson's series inequalities by replacing

doi:10.5186/aasfm.2014.3947

2010 Mathematics Subject Classification: Primary 26D15; Secondary 42B25.

Key words: Hardy inequalities, Muckenhoupt weights. 
means by more general linear transforms. For the study of Copson's inequality one can also see [3]. Additionally, in [4] Elliot has already proved inequality (1.2) by similar methods to those that appear in [2].

There is a continued analogue of Theorem 1.1 (see [8]) which can be stated as

Theorem C. If $p>1, f(x) \geq 0$ for $x \in[0,+\infty)$, then

$$
\int_{0}^{\infty}\left(\frac{1}{x} \int_{0}^{x} f(t) d t\right)^{p} d x<\left(\frac{p}{p-1}\right)^{p} \int_{0}^{\infty} f^{p}(x) d x
$$

Further generalizations of (1.3) can be seen in [6]. Other authors have also studied these inequalities in more general forms as it may be seen in [13] and [17]. Landau has also studied the above inequality and his work appears in [11]. For a complete discussion of the topic one can consult [10] and [16].

There is an analogue of (1.3) for negative exponents which is presented in [9] without proof. This is the following

Theorem D. Let $f:[a, b] \rightarrow \mathbf{R}^{+}$. Then the following is true when every $p$ is positive

$$
\int_{a}^{b}\left(\frac{1}{x-a} \int_{a}^{x} f(y) d y\right)^{-p} d x \leq\left(\frac{p+1}{p}\right)^{p} \int_{a}^{b} f^{-p}(x) d x,
$$

Moreover, (1.4) is best possible.

In this paper we generalize (1.4) by proving the following

Theorem 1. Let $p \geq q>0$ and $f:[a, b] \rightarrow \mathbf{R}^{+}$. The following inequality is true and sharp

$$
\int_{a}^{b}\left(\frac{1}{x-a} \int_{a}^{x} f(y) d y\right)^{-p} d x \leq\left(\frac{p+1}{p}\right)^{q} \int_{a}^{b}\left(\frac{1}{x-a} \int_{a}^{x} f(y) d y\right)^{-p+q} f^{-q}(x) d x
$$

In fact more is true as can be seen in

Theorem 2. Let $p \geq q>0$ and $a_{n} \geq 0, \lambda_{n}>0$ for $n=1,2, \ldots$. Define $A_{n}$ and $\Lambda_{n}$ as in Theorem B. Then

$$
\sum_{n=1}^{\infty} \lambda_{n}\left(\frac{A_{n}}{\Lambda_{n}}\right)^{-p} \leq\left(\frac{p+1}{p}\right)^{q} \sum_{n=1}^{\infty}\left(\frac{A_{n}}{\Lambda_{n}}\right)^{-p+q} a_{n}^{-q} .
$$

Theorem 2 implies easily Theorem 1 , by setting $\lambda_{n}=1$, for every $n \in \mathbf{N}$, and by using an approximation argument of any $f$ by simple functions on $[a, b]$.

We believe that the above two theorems should have many applications especially in the theory of weights and other fields. In this paper we give such an application of Theorem 1. More precisely we give a proof of a result that appears in [9] based on that in [1]. This is described as follows:

Let $f:[0,1) \rightarrow \mathbf{R}^{+}$be non-decreasing such that it satisfies the $A_{q}$ condition for some $q>1$ upon all subintervals of $[0,1]$ with constant $M \geq 1$. That is the following hold:

$$
\left(\frac{1}{b-a} \int_{a}^{b} f(y) d y\right)\left(\frac{1}{b-a} \int_{a}^{b} f^{-1 /(q-1)}(y) d y\right)^{q-1} \leq M
$$

for every $(a, b) \subseteq[0,1]$. 
Let now $p_{0} \in[1, q]$ be defined as the solution of the following equality

$$
\frac{q-p_{0}}{q-1}\left(M p_{0}\right)^{1 /(q-1)}=1
$$

We want to describe the $A_{p}$ properties of $f$ for any $p<q$. This is proved in [3]. More precisely the following is true:

Theorem E. Let $f:[0,1) \rightarrow \mathbf{R}^{+}$be non-decreasing satisfying (1.7). Then for every $p \in\left(p_{0}, q\right]$ we have that $f \in L^{-1 /(p-1)}([0,1))$, where $p_{0}$ is defined by (1.8). Moreover, the following inequality is true

$$
\left(\frac{1}{b-a} \int_{a}^{b} f(y) d y\right)\left(\frac{1}{b-a} \int_{a}^{b} f^{-1 /(p-1)}(y) d y\right)^{p-1} \leq M^{\prime}
$$

for any $(a, b) \subseteq[0,1], p \in\left(p_{0}, q\right]$ where $M^{\prime}=M^{\prime}(p, q, M)$. Additionally, the result is best possible. That is, we cannot decrease $p_{0}$.

To be more precise we are interested in those $p$ such that $1<p \leq q$ for which $f \in L^{-1 /(p-1)}([0,1))$ whenever $f$ satisfies $(1.7)$ for some $M \geq 1$. In fact this is equivalent to an inequality of the form of (1.9) for every such $p$. The exact best possible range of those $p$ is provided by the above theorem. Our aim in this paper is to provide an alternative proof of the above fact by proving the following:

Theorem 3. Let $f:[0,1) \rightarrow \mathbf{R}^{+}$be non-decreasing satisfying (1.7) for all subintervals of the form $(0, t), t \in(0,1]$. That is, the following hold:

$$
\left(\frac{1}{t} \int_{0}^{t} f(y) d y\right)\left(\frac{1}{t} \int_{0}^{t} f^{-1 /(q-1)}(y) d y\right)^{q-1} \leq M
$$

for any $t \in(0,1]$. Then, the following is true: For any $p \in\left(p_{0}, q\right]$, where $p_{0}$ is defined by (1.8), there exists $M^{\prime}=M^{\prime}(p, q, M)$ such that

$$
\left(\frac{1}{t} \int_{0}^{t} f(y) d y\right)\left(\frac{1}{t} \int_{0}^{t} f^{-1 /(p-1)}(y) d y\right)^{p-1} \leq M^{\prime}, \text { for every } t \in(0,1]
$$

Additionally, the result is best possible.

The analogue then of Theorem 3 for the class of intervals of the form $(t, 1]$, $t \in[0,1)$ can be proved in a similar way. The following now is true as can be seen in [9].

Theorem F. If $f:[0,1) \rightarrow \mathbf{R}^{+}$satisfies (1.7) upon all subintervals of $[0,1)$ of the form $(0, t)$ and $(t, 1)$, for $t \in(0,1]$, and if additionally $f$ is monotone then (1.7) is implied for the class of all subintervals $(a, b) \subseteq[0,1)$.

Thus Theorem 3 and it's analogue that was mentioned above imply Theorem E.

The paper is organized as follows: In Section 2 we prove Theorem 2 and a generalization of it named as Theorem 4, while in Section 3 we prove the application mentioned above. 


\section{The Hardy inequality}

Proof of Theorem 2. Let $a_{n}, \lambda_{n}>0$ for every $n=1,2, \ldots$ We are going to prove for every $N \in \mathbf{N}, p>0$ and $q \in(0, p]$ that the following inequality holds

$$
\sum_{n=1}^{N} \lambda_{n}\left(\frac{A_{n}}{\Lambda_{n}}\right)^{-p} \leq\left(\frac{p+1}{p}\right)^{q} \sum_{n=1}^{N} \lambda_{n}\left(\frac{A_{n}}{\Lambda_{n}}\right)^{-p+q} a_{n}^{-q} .
$$

We will use the following well known elementary inequality

$$
p y^{p+1}-(p+1) y^{p} \geq-1,
$$

for every $u \geq 0$ and $p>0$.

For its proof we consider the function $F(y)=p y^{p+1}-(p+1) y^{p}$, for $y \geq 0$ and find easily that its minimum is attained for $y=1$. From (2.2) we deduce that

$$
y^{-p}+p y \geq p+1, \text { for any } y, p>0 .
$$

We apply the last inequality for $y=y_{1} / y_{2}$, thus

$$
y_{1}^{-p}+p y_{1} y_{2}^{-p-1}-(p+1) y_{2}^{-p} \geq 0,
$$

whenever $y_{1}, y_{2}>0$. For any fixed $n \in \mathbf{N}$ we define

$$
y_{1}=\left(\frac{p}{p+1}\right)^{1+q / p} \cdot a_{n}^{q / p} \cdot\left(\frac{A_{n}}{\Lambda_{n}}\right)^{1-q / p}, \quad y_{2}=\left(\frac{p}{p+1}\right) \frac{A_{n}}{\Lambda_{n}} .
$$

Then

$$
\begin{aligned}
y_{2}^{-p} & =\left(\frac{p}{p+1}\right)^{-p}\left(\frac{A_{n}}{\Lambda_{n}}\right)^{-p}, y_{1}^{-p}=\left(\frac{p}{p+1}\right)^{-p-q} a_{n}^{-q}\left(\frac{A_{n}}{\Lambda_{n}}\right)^{-p+q}, \text { and } \\
y_{1} y_{2}^{-p-1} & =\left(\frac{p}{p+1}\right)^{-p+q / p} a_{n}^{q / p} \cdot\left(\frac{A_{n}}{\Lambda_{n}}\right)^{-p-q / p} .
\end{aligned}
$$

Thus from (2.3) we have that

$$
\begin{aligned}
& \left(\frac{p}{p+1}\right)^{-p-q} a_{n}^{-q}\left(\frac{A_{n}}{\Lambda_{n}}\right)^{-p+q}+p\left(\frac{p}{p+1}\right)^{-p+q / p} a_{n}^{q / p}\left(\frac{A_{n}}{\Lambda_{n}}\right)^{-p-q / p} \\
& -(p+1)\left(\frac{p}{p+1}\right)^{-p}\left(\frac{A_{n}}{\Lambda_{n}}\right)^{-p} \geq 0 \Longrightarrow \\
& \left(\frac{p+1}{p}\right)^{q} a_{n}^{-q}\left(\frac{A_{n}}{\Lambda_{n}}\right)^{-p+q}+p\left(\frac{p}{p+1}\right)^{q / p} a_{n}^{q / p}\left(\frac{A_{n}}{\Lambda_{n}}\right)^{-p-q / p} \geq(p+1)\left(\frac{A_{n}}{\Lambda_{n}}\right)^{-p}
\end{aligned}
$$

We multiply (2.4) by $\lambda_{n}$ and sum the respective inequalities for $n=1,2, \ldots, N$. As a result we obtain the following

$$
\begin{aligned}
& \left(\frac{p+1}{p}\right)^{q} \sum_{n=1}^{N} \lambda_{n} a_{n}^{-q}\left(\frac{A_{n}}{\Lambda_{n}}\right)^{-p+q}+p\left(\frac{p+1}{p}\right)^{-q / p} \sum_{n=1}^{N} \lambda_{n} a_{n}^{q / p}\left(\frac{A_{n}}{\Lambda_{n}}\right)^{-p-q / p} \\
& \geq(p+1) \sum_{n=1}^{N} \lambda_{n}\left(\frac{A_{n}}{\Lambda_{n}}\right)^{-p} .
\end{aligned}
$$


Suppose now that we have shown that

$$
\sum_{n=1}^{N} \lambda_{n} a_{n}^{q / p}\left(\frac{A_{n}}{\Lambda_{n}}\right)^{-p-q / p} \leq\left(\frac{p+1}{p}\right)^{q / p} \sum_{n=1}^{N} \lambda_{n}\left(\frac{A_{n}}{\Lambda_{n}}\right)^{-p}
$$

Then immediately from (2.5) and (2.6) we conclude (2.1). Thus we just need to prove the following inequality

$$
\sum_{n=1}^{N} \lambda_{n} a_{n}^{\varepsilon}\left(\frac{A_{n}}{A_{n}}\right)^{-p-\varepsilon} \leq\left(\frac{p+1}{p}\right)^{\varepsilon} \sum_{n=1}^{N} \lambda_{n}\left(\frac{A_{n}}{\Lambda_{n}}\right)^{-p},
$$

for any $\varepsilon \in(0,1]$.

We first prove $(2.7)$ for $\varepsilon=1$. We state it as

Lemma 2.1. Let $a_{n}, \lambda_{n}>0$, for $n=1,2, \ldots$ and $A_{n}, \Lambda_{n}$ defined as above. Then the following inequality is true for any $N \in \mathbf{N}$

$$
\sum_{n=1}^{N} \lambda_{n} a_{n}\left(\frac{A_{n}}{\Lambda_{n}}\right)^{-p-1} \leq\left(\frac{p+1}{p}\right) \sum_{n=1}^{N} \lambda_{n}\left(\frac{A_{n}}{\Lambda_{n}}\right)^{-p} .
$$

Proof. We prove inductively the following inequality

$$
\sum_{n=1}^{N} \lambda_{n}\left(\frac{A_{n}}{\Lambda_{n}}\right)^{-p}-\left(\frac{p}{p+1}\right) \sum_{n=1}^{N} \lambda_{n} a_{n}\left(\frac{A_{n}}{\Lambda_{n}}\right)^{-p-1} \geq \frac{\Lambda_{N}}{p+1}\left(\frac{A_{N}}{\Lambda_{N}}\right)^{-p}
$$

For $N=1(2.8)$ is obviously an equality.

Let us suppose that (2.8) is true with $N-1$, in place of $N$. Then we define

$$
\begin{aligned}
S_{N}= & \sum_{n=1}^{N}\left[\lambda_{n}\left(\frac{A_{n}}{\Lambda_{n}}\right)^{-p}-\left(\frac{p}{p+1}\right) \lambda_{n} a_{n}\left(\frac{A_{n}}{\Lambda_{n}}\right)^{-p-1}\right] \\
= & \sum_{n=1}^{N-1}\left[\lambda_{n}\left(\frac{A_{n}}{\Lambda_{n}}\right)^{-p}-\left(\frac{p}{p+1}\right) \lambda_{n} a_{n}\left(\frac{A_{n}}{\Lambda_{n}}\right)^{-p-1}\right] \\
& +\lambda_{N}\left(\frac{A_{N}}{\Lambda_{N}}\right)^{-p}-\left(\frac{p}{p+1}\right)\left(A_{N}-A_{N-1}\right)\left(\frac{A_{N}}{\Lambda_{N}}\right)^{-p-1} .
\end{aligned}
$$

Using the induction step (2.9) becomes

$$
\begin{aligned}
S_{N} \geq & \frac{\Lambda_{N-1}}{p+1}\left(\frac{A_{N-1}}{\Lambda_{N-1}}\right)^{-p}+\lambda_{N}\left(\frac{A_{N}}{\Lambda_{N}}\right)^{-p}-\left(\frac{p}{p+1}\right)\left(A_{N}-A_{N-1}\right)\left(\frac{A_{N}}{\Lambda_{N}}\right)^{-p-1} \\
= & \frac{\Lambda_{N-1}}{p+1}\left(\frac{A_{N-1}}{\Lambda_{N-1}}\right)^{-p}+\lambda_{N} \cdot\left(\frac{A_{N}}{\Lambda_{N}}\right)^{-p}-\frac{p}{p+1} \Lambda_{N} \cdot\left(\frac{A_{N}}{\Lambda_{N}}\right)^{-p} \\
& +\frac{\Lambda_{N-1}}{p+1} \cdot p \cdot \frac{A_{N-1}}{\Lambda_{N-1}}\left(\frac{A_{N}}{\Lambda_{N}}\right)^{-p-1} .
\end{aligned}
$$


Using now inequality (2.3) in the last term in (2.10) we conclude that

$$
\begin{aligned}
S_{N} \geq & \frac{\Lambda_{N-1}}{p+1}\left(\frac{A_{N-1}}{\Lambda_{N-1}}\right)^{-p}+\lambda_{N}\left(\frac{A_{N}}{\Lambda_{N}}\right)^{-p}-\frac{p}{p+1} \Lambda_{N}\left(\frac{A_{N}}{\Lambda_{N}}\right)^{-p} \\
& +\frac{\Lambda_{N-1}}{p+1}\left((p+1)\left(\frac{A_{N}}{\Lambda_{N}}\right)^{-p}-\left(\frac{A_{N-1}}{\Lambda_{N-1}}\right)^{-p}\right) \\
= & \left(\frac{A_{N}}{\Lambda_{N}}\right)^{-p}\left(\lambda_{N}-\frac{p}{p+1} \Lambda_{N}+\Lambda_{N-1}\right)=\frac{\Lambda_{N}}{p+1}\left(\frac{A_{N}}{\Lambda_{N}}\right)^{-p} .
\end{aligned}
$$

Inequality (2.8) is proved.

We now prove inequality (2.6). If we fix $q \in(0, p)$, then using Lemma 2.1 and applying Holder's inequality with the exponents $r=\frac{p}{q}$ and $r^{\prime}=\frac{r}{r-1}=\frac{p}{p-q}$, we get

$$
\begin{aligned}
\sum_{n=1}^{N} \lambda_{n} a_{n}^{q / p}\left(\frac{A_{n}}{\Lambda_{n}}\right)^{-q-q / p}\left(\frac{A_{n}}{\Lambda_{n}}\right)^{-p+q} & \leq\left\{\sum_{n=1}^{N} \lambda_{n} a_{n}\left(\frac{A_{n}}{\Lambda_{n}}\right)^{-p-1}\right\}^{q / p}\left\{\sum_{n=1}^{N} \lambda_{n}\left(\frac{A_{n}}{\Lambda_{n}}\right)^{-p}\right\}^{1-q / p} \\
& \leq\left(\frac{p+1}{p}\right)^{q} \sum_{n=1}^{N} \lambda_{n}\left(\frac{A_{n}}{\Lambda_{n}}\right)^{-p} .
\end{aligned}
$$

In this way we derived the proof of equality (2.6). The proof of Theorem 2 is now complete.

We state now the following as

Corollary 2.1. If $\left(a_{n}\right)_{n}$ is a sequence of positive real numbers and $p>0$, then for every $q \in(0, p]$, the following inequality is true

$$
\sum_{n=1}^{\infty}\left(\frac{1}{n} \sum_{k=1}^{n} a_{k}\right)^{-p} \leq\left(\frac{p+1}{p}\right)^{q}\left(\sum_{k=1}^{\infty} a_{k}\right)^{-p+q} a_{n}^{-q} .
$$

Proof. Immediate from Theorem 2, if we set $\lambda_{n}=1$ for every $n \in \mathbf{N}$.

From Corollary 2.1 and a standard approximation argument we obtain as a consequence Theorem 1. It's sharpness is easily verified and is proved as the sharpness of (1.2). For it's proof we just need to consider functions of the form $f(x)=(x-a)^{d}$, with $d \rightarrow \frac{1^{-}}{p}$. Then the fraction of the integrals in (1.2) tends to the constant $\left(\frac{p+1}{p}\right)^{q}$

Before we end this section we will give another one

Theorem 4. Let $a_{n}, \lambda_{n}>0$ and $A_{n}, \Lambda_{n}$ defined as in Theorem 2. Then for every $0<q_{1} \leq q_{2} \leq p$ the following inequality holds

$$
\sum_{n=1}^{\infty} \lambda_{n}\left(\frac{A_{n}}{\Lambda_{n}}\right)^{-p+q_{1}} a_{n}^{q_{1}} \leq\left(\frac{p+1}{p}\right)^{q_{2}-q_{1}} \sum_{n=1}^{\infty} \lambda_{n}\left(\frac{A_{n}}{\Lambda_{n}}\right)^{-p+q_{2}} a_{n}^{-q_{2}} .
$$


Proof. Fix $N \in \mathbf{N}$. As in Lemma 1 we set for any $q \in[0, p]$,

$$
J_{q}=\sum_{n=1}^{N} \lambda_{n}\left(\frac{A_{n}}{\Lambda_{n}}\right)^{-p+q} a_{n}^{-q} .
$$

Then using Hölder's inequality and Theorem 2, we obtain

$$
J_{q_{1}} \leq J_{q_{2}}^{q_{1} / q_{2}} \cdot J_{0}^{1-q_{1} / q_{2}} \leq\left(\frac{p+1}{p}\right)^{q_{2}-q_{1}} J_{q_{2}} .
$$

So the proof of inequality (2.11) is complete.

\section{Muckenhoupt weights on $R$}

We will give now an application of the results in Section 2. More precisely we will give the proof of Theorem 3. For this purpose we will use the following

Lemma 3.1. Let $\psi:(0,1] \rightarrow[0, \infty)$ such that $\lim _{t \rightarrow 0} t \cdot \psi(t)^{a}=0$, where $a$ is a real constant greater than 1 and $\psi(t)$ is a function that is continuous and monotone on $(0,1]$. Then the following is true for any $u \in(0,1]$ :

$$
a \int_{0}^{u} \psi^{a-1}(t)(t \cdot \psi(t))^{\prime} d t=u \psi^{a}(u)+(a-1) \int_{0}^{u} \psi^{a}(t) d t .
$$

Proof. By our hypothesis the following integration by parts formula holds

$$
a \int_{0}^{u} t \psi^{a-1}(t) \psi^{\prime}(t) d t=u \psi^{a}(u)-\int_{0}^{u} \psi^{a}(t) d t
$$

We obtain the required identity now, by adding $a \int_{0}^{u} \psi^{a}(t) d t$ to both sides of the above equation.

We are now ready to continue with the

Proof of Theorem 3. Let $f:[0,1) \rightarrow \mathbf{R}^{+}$be non-decreasing which satisfies the following inequality

$$
\left(\frac{1}{t} \int_{0}^{t} f\right)\left(\frac{1}{t} \int_{0}^{t} f^{-1 /(q-1)}\right)^{q-1} \leq M,
$$

for any $t \in(0,1]$, where $q$ is fixed such that $q>1$.

Additionally, we suppose that there exists a constant $\varepsilon=\varepsilon_{f}>0$ such that $f(t) \geq \varepsilon, \forall t \in[0,1)$. We define now the following function $h:[0,1) \rightarrow \mathbf{R}^{+}$by $h(t)=f^{-1 /(q-1)}(t)$, for any $t \in[0,1)$. Thus, $h$ is bounded on $[0,1)$ by $\varepsilon^{-1 /(q-1)}$.

We apply now Lemma 3.1 for $a=\frac{q-1}{p-1}$, which is greater than 1 whenever $p \in[1, q)$ and for $\psi$ defined by: $\psi(t)=\frac{1}{t} \int_{0}^{t} f^{-1 /(q-1)}$. Note that, since $f$ is nondecreasing and $h$ is bounded above the hypothesis of Lemma 3.1 are satisfied. As a consequence we have the following identity

$$
\begin{aligned}
& \frac{q-1}{q-p} \int_{0}^{t} f^{-1 /(q-1)}(s)\left(\frac{1}{s} \int_{0}^{s} f^{-1 /(q-1)}\right)^{(q-p) /(p-1)} d s-\int_{0}^{t}\left(\frac{1}{s} \int_{0}^{s} f^{-1 /(q-1)}\right)^{(q-1) /(p-1)} d s \\
& =\frac{p-1}{q-p} \frac{1}{t^{(q-p) /(p-1)}}\left(\int_{0}^{t} f^{-1 /(q-1)}\right)^{(q-1) /(p-1)} .
\end{aligned}
$$


Moreover, we define the following function $h_{y}(x)$ with variable $x$, for any constant $y>0$ by

$$
h_{y}(x)=\frac{q-1}{q-p} y x^{(q-p) /(p-1)}-x^{(q-1) /(p-1)}, \text { for } x \geq y .
$$

Then $h_{y}^{\prime}(x)=\frac{q-1}{p-1} x^{(q-1) /(p-1)-1}(y-x) \leq 0$, for any $x \geq y$. Thus $h_{y}$ is decreasing on $[y,+\infty)$. So if $y \leq x \leq w$ we must have that $h_{y}(x) \geq h_{y}(w)$. We set now for any $s \in(0, t]$

$$
x=\frac{1}{s} \int_{0}^{s} f^{-1 /(q-1)}, \quad y=f^{-1 /(q-1)}(s), \quad c=M^{1 /(q-1)}, \quad z=\left(\frac{1}{s} \int_{0}^{s} f\right)^{-1 /(q-1)} .
$$

Then, by our hypothesis we have that $y \leq x \leq w=c z$, since $f$ is non-decreasing and (1.10) is satisfied for $f$. Thus $h_{y}(x) \geq h_{y}(w)$, that is

$$
\begin{aligned}
& \frac{q-1}{q-p} f^{-1 /(q-1)}(s)\left(\frac{1}{s} \int_{0}^{s} f^{-1 /(q-1)}\right)^{(q-p) /(p-1)}-\left(\frac{1}{s} \int_{0}^{s} f^{-1 /(q-1)}\right)^{(q-1) /(p-1)} \\
& \geq \frac{q-1}{q-p} f^{-1 /(q-1)}(s)\left(\frac{1}{s} \int_{0}^{s} f\right)^{-1 /(p-1)+1 /(q-1)} c^{q-p /(p-1)}-c^{(q-1) /(p-1)}\left(\frac{1}{s} \int_{0}^{s} f\right)^{-1 /(p-1)} .
\end{aligned}
$$

Integrating the inequality just mentioned over $(0, t)$ and using the equality that is presented above we have after canceling a suitable power of $c$, the following inequality

$$
\begin{aligned}
& \frac{q-1}{q-p} \int_{0}^{t} f^{-1 /(q-1)}(s)\left(\frac{1}{s} \int_{0}^{s} f\right)^{-1 /(p-1)+1 /(q-1)} d s \\
& \leq c \int_{0}^{t}\left(\frac{1}{s} \int_{0}^{s} f\right)^{-1 /(p-1)} d s+\frac{p-1}{q-p} M^{1 /(p-1)} t\left(\frac{1}{t} \int_{0}^{t} f\right)^{-1 /(p-1)} \cdot \frac{1}{c^{(q-p) /(p-1)}}
\end{aligned}
$$

We use now Theorem 1 with $\frac{1}{p-1}$ in place of $p$ and $\frac{1}{q-1}$ in place of $q$, so we have that

$$
\int_{0}^{t}\left(\frac{1}{s} \int_{0}^{s} f\right)^{-1 /(p-1)} d s \leq p^{1 /(q-1)} \int_{0}^{t}\left(\frac{1}{s} \int_{0}^{s} f\right)^{-1 /(p-1)+1 /(q-1)} f^{-1 /(q-1)} d s .
$$

Combining now (3.1) and (3.2) we see immediately that

$$
\begin{aligned}
& {\left[1-p^{1 /(q-1)} \frac{q-p}{q-1} c\right] \frac{1}{t} \int_{0}^{t} f^{-1 /(q-1)}(s)\left(\frac{1}{s} \int_{0}^{s} f\right)^{-1 /(p-1)+1 /(q-1)} d s} \\
& \leq M^{1 /(p-1)} \frac{p-1}{q-1} \frac{1}{c^{(q-p) /(p-1)}}\left(\frac{1}{t} \int_{0}^{t} f\right)^{-1 /(p-1)} .
\end{aligned}
$$

If we restrict now $p$ to the interval $\left(p_{0}, q\right]$ where $p_{0} \in[1, q]$ is the unique root of the equation $\frac{q-p_{0}}{q-1}\left(M p_{0}\right)^{1 /(q-1)}=1$, we must have that for such $p$ the following constant $K=K(p, q, c)=1-p^{1 /(q-1)} \frac{q-p}{q-1} \cdot c$ is positive, and if we note that

$$
\left(\frac{1}{s} \int_{0}^{s} f\right)^{-1 /(p-1)+1 /(q-1)} \geq f^{-1 /(p-1)+1 /(q-1)}(s)
$$

which is true since $p<q$ and $f$ is non-decreasing, we must have by (3.3) that

$$
\frac{1}{t} \int_{0}^{t} f^{-1 /(p-1)}(s) d s \leq \Lambda\left(\frac{1}{t} \int_{0}^{t} f\right)^{-1 /(p-1)}
$$


where $\Lambda=\Lambda(p, q, c)$ is a positive real constant.

In this way we derived our result, for functions $f:[0,1) \rightarrow \mathbf{R}^{+}$bounded below by a constant $\varepsilon>0$. A truncation argument give the result for arbitrary $f$.

At last we need to prove that our result is sharp. We search for a function of the form

$$
f(t)=t^{a}, \text { with } 0<a<q-1 .
$$

For any such $a$ we have that

$$
\left(\frac{1}{t} \int_{0}^{t} f\right)\left(\frac{1}{t} \int_{0}^{t} f^{-1 /(q-1)}\right)=\frac{1}{a+1}\left(\frac{p-1}{q-1-a}\right)^{q-1}=M(q, a)
$$

for any $t \in(0,1]$, as can be easily seen.

Thus, $f$ satisfies the $A_{q}$ condition for any $q>a+1$. If we set $a=p_{0}-1$, where $p_{0}$ is defined as above we have that $f$ satisfies the $A_{p}$ condition for any $p>p_{0}$, while for $p=p_{0}$ it is no longer satisfied. Thus our theorem is sharp and by this way we end it's proof.

\section{References}

[1] D'Appuzzo, L., and C. Sbordone: Reverse Hölder inequalities. A sharp result. - Rend. Mat. Appl. (7) 10, 1990, 357-366.

[2] Copson, E. T.: Note on series of positive terms. - J. London Math. Soc. 2, 1927, 9-12, and 3, $1928,49-51$.

[3] Copson, E. T.: Some integral inequalities. - Proc. Roy. Soc. Edinburgh Sect. A 75, 1975/1976, $157-164$.

[4] Elliot, E. B.: A simple exposition of some recently proved facts as to convergency. - J. London Math. Soc. 1, 1926, 93-96.

[5] Hardy, G. H.: Note on a theorem of Hilbert. - Math Z. 6, 1920, 314-317.

[6] Hardy, G. H.: Notes on some points in the integral calculus, L. X. An inequality between integrals. - Messenger of Math. 57, 1928, 12-16.

[7] Hardy, G. H., and J. E. Littlewood: Elementary theorems concerning power series with positive coefficients and moment constants of positive functions. - J. Reine Angew. Math. 157, $1927,141-158$.

[8] Hardy, G. H., J. E. Littlewood, and G. Polya: Inequalities. - Cambridge Univ. Press, Cambridge, 1934.

[9] KonenovskiI, A. A.: The exact continuation of a Reverse Hölder inequality and Muckenhoupt's conditions. - Math. Notes 52, 1992, 1192-1201.

[10] Kufner, A., L. Maligranda, and L. E. Persson: The prehistory of the Hardy inequality. - Amer. Math. Monthly 113:8, 2006, 715-732.

[11] Landau, E.: A note on a theorem concerning series of positive terms: Extract from a letter of Prof. E. Landau to Prof. I. Schur. - J. London Math. Soc. 1, 1926, 38-39.

[12] Leindler, L.: Generalization of inequalities of Hardy and Littlewood. - Acta Sci. Math. (Szeged) 31, 1970, 279-285.

[13] Levinson, N.: Generalizations of an inequality of Hardy. - Duke Math. J. 31, 1964, 389-394.

[14] Love, E. R.: Generalizations of Hardy's and Copson's inequalities. - J. London Math. Soc. $30,1984,431-440$.

[15] Nemeth, J.: Generalizations of the Hardy-Littlewood inequality. - Acta Sci. Math. (Szeged) $32,1971,295-299$. 
[16] Pachpatte, B. G.: Mathematical inequalities. - North-Holland Math. Library 67, 2005.

[17] Pachpatte, B. G.: On a new class of Hardy type inequalities. - Proc. Roy. Soc. Edinburgh Sect. A 105, 1987, 265-274.

Received 23 December 2013 • Accepted 11 April 2014 